\title{
RF AND DATA ACQUISITION SYSTEMS FOR FERMILAB'S ILC SRF CAVITY VERTICAL TEST STAND*
}

\author{
Joseph P. Ozelis" ${ }^{\#}$, Roger Nehring, Fermilab, Batavia, IL. 60510, U.S.A. \\ Christiana Grenoble, Thomas J. Powers, TJNAF, Newport News, VA. 23696, U.S.A.
}

\begin{abstract}
Fermilab is developing a facility for vertical testing of SRF cavities as part of its ILC program. The RF system for this facility is based on the proven production cavity test systems used at Jefferson Lab for CEBAF and SNS cavity testing. The design approach is modular in nature, using commercial-off-the-shelf (COTS) components. This yields a system that can be easily debugged and modified, and with ready availability of spares. Comprehensive data acquisition and control is provided by a PXI-based hardware platform in conjunction with software developed in the LabView programming environment.
\end{abstract}

\section{INTRODUCTION}

The general approach to the architecture and overall layout of the RF system to be used for testing superconducting cavities in the vertical test stand at Fermilab[1] has been to construct a system that is modular in nature and based almost exclusively on readily available commercial components. This, together with basing the design upon a well-established successful system at Jefferson Lab[2], ensured that a system could be fabricated, installed, and commissioned with a minimal amount of development and risk, and could take advantage of existing experience and expertise in SRF cavity testing at FNAL and JLab, as well as JLab's LabView-based software tools. Such a system is also well suited to the production-scale testing that will be required as part of the ILC program.

\section{SYSTEM REQUIREMENTS}

In order to characterize the performance of an SRF cavity, one must simultaneously determine the accelerating gradient and unloaded cavity quality factor $\mathrm{Q}_{0}$. The presence and magnitude of any $\gamma$ radiation produced as a result of field emission must also be recorded, to aid in understanding the nature of cavity performance limitations.

The cavity gradient, $\mathrm{Q}_{0}$, and the field probe $\mathrm{Q}\left(\mathrm{Q}_{\mathrm{fp}}\right)$ can be calculated by determining the decay constant of the cavity field after the RF power is switched off, and measuring the $\mathrm{CW}$ incident, reflected and transmitted power signals. Once the external-Q of the field probe $\left(\mathrm{Q}_{\mathrm{fp}}\right)$ has been calculated, $\mathrm{E}$ and $\mathrm{Q}_{0}$ can thereafter be calculated solely by using the incident, forward and transmitted power signals[3]. The required measurement parameters for these two methods of determining cavity $\mathrm{E}$ and $\mathrm{Q}_{0}$, are $\mathrm{P}_{\mathrm{i}}, \mathrm{P}_{\mathrm{r}}, \mathrm{P}_{\mathrm{t}}, \mathrm{P}_{\text {HOM-A }}, \mathrm{P}_{\text {HOM-B }}, \tau$, and $\mathrm{f}$.

\footnotetext{
*Work supported by U.S. Department of Energy under contract DEAC02-76CH03000

\#ozelis@fnal.gov
}

$\mathrm{P}_{\mathrm{i}}, \mathrm{P}_{\mathrm{r}}$, and $\mathrm{P}_{\mathrm{t}}$ are the average incident, reflected, and transmitted power to/from the cavity, $\tau$ is the cavity field decay time constant (used only in the decay mode), and $\mathrm{f}$ is the cavity frequency (only used in CW mode to measure Lorentz force detuning). If the cavity is equipped with HOM filters and couplers, then the transmitted power from these couplers $\left(\mathrm{P}_{\mathrm{HOM}-\mathrm{A}}, \mathrm{P}_{\mathrm{HOM}-\mathrm{B}}\right)$ must also be measured in order to correctly calculate the cavity power dissipation.

These quantities are measured using power meters, crystal detectors (for cavity transmitted power decay time measurement and initial determination of coupling sign), and a frequency counter. In order to keep the error on the decay time constant measurement and determination of $\mathrm{Q}_{\mathrm{FP}}$ low ( 2\%), the crystal detector must operate in the square-law regime, requiring that its input be appropriately attenuated when necessary. Radiation produced by field emitted electrons is measured using a Canberra GP110M probe, in conjunction with the Canberra ADM606M probe power supply and readout. This commercially available system was interfaced with the data acquisition platform to record field emission data.

\section{SYSTEM HARDWARE COMPONENTS}

The chosen architecture provides the essential RF control for the test stand by using the classic combination of oscillator, phase detector/mixer, and loop amplifier to detect the resonant cavity frequency and adjust the RF source frequency to lock onto the cavity. The phase difference between transmitted power and drive signals is converted by the mixer to a signal that is used to adjust the RF source frequency. A simplified block diagram of the system is shown in Figure 1.

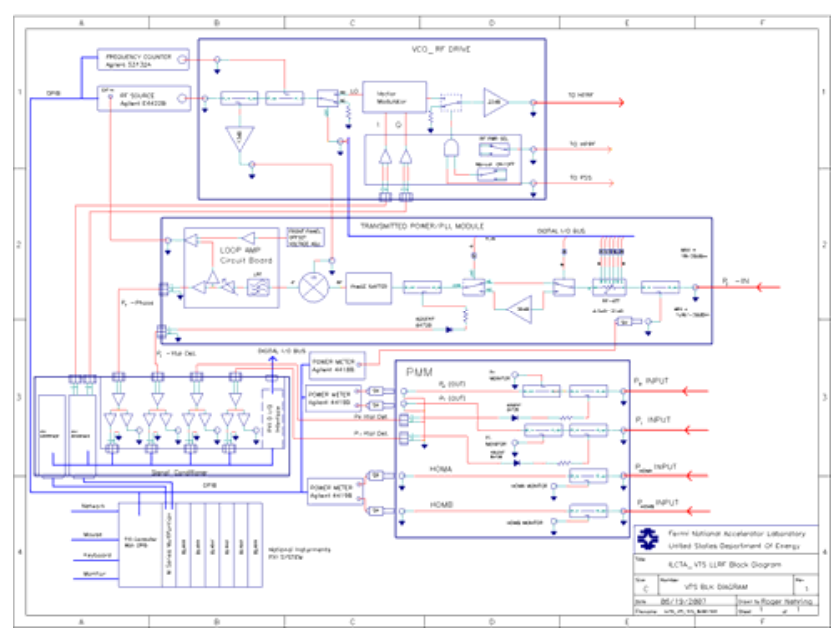

Figure 1. Block diagram of LLRF system. 
The system consists of the following components and/or functional modules:

- RF Source Module

- PLL/Transmitted Power Network

- Power Measurement Module

- High Power Amplifier Switching Network

- Signal Conditioning Module

- Control and Data Acquisition Platform

\section{RF Source Module}

The RF Source Module contains an RF source (Agilent E4422B) that functions as a VCO, supporting an adjustable FM (frequency modulation) range. The output signal of the source is input to a vector modulator that provides an attenuation range of $60 \mathrm{~dB}$ and a phase adjustment of $360^{\circ}$. The output of the source can be modulated using a GaAs FET switch to provide a low rate pulse capability, useful in cavity field emitter processing. The output of the vector modulator passes through a manual SPST switch which is part of a logic circuit and allows the user to enable RF. This logic circuit is coupled to the Personnel Safety System interlock, and requires the presence of an RF enable signal from the interlocks.

\section{PLL/Transmitted Power Network}

The PLL/Transmitted Power Network module conditions and amplifies the transmitted power signal from the SRF cavity. This signal passes through a digital attenuator and optionally through a $27 \mathrm{~dB}$ low noise amplifier. The attenuator provides $30.5 \mathrm{~dB}$ of attenuation with $0.5 \mathrm{~dB}$ (6 bit) resolution and $<10^{\circ}$ phase error. Residual phase error is compensated by using a phase error look-up table in the control software. The combination of attenuator and LNA accommodates the approximately $60 \mathrm{~dB}$ dynamic range of the transmitted power signal that results both from the expected range in external Q of the field probe and cavity field.

The transmitted power signal is coupled to the RF input of a double balanced mixer, while a portion of the output from the RF source provides the LO input for the mixer. The output of the mixer yields a signal proportional to the product of the phase error and field probe voltage, which is then amplified by a variable-gain (1-800) loop amplifier. The DC voltage of the loop amplifier serves as the FM input for the RF source. A pair of 10-turn potentiometers provide coarse and fine adjustment of the offset for this voltage and hence the output frequency of the RF source which, along with a manual phase shifter providing $180^{\circ} / \mathrm{GHz}$ phase shift, is particularly useful in initial tuning of the loop to a new cavity.

\section{Power Measurement Module}

The power measurement module (PMM) provides a convenient means for interfacing to the RF power meters. A $20 \mathrm{~dB}$ dual directional coupler on the output of the high power amplifier switching network provides signals for the reflected and incident RF power, which are routed directly to the PMM, and are interfaced with the power meter sensors and power meters with suitable in-line attenuators. Wide dynamic range power sensors are necessary to ensure a low enough noise floor for accurate calibration at low power while having sufficient power margin for high power system operation. The signals from the cavity's HOM filter pickup probes are also routed to this module, and interfaced with a similar set of power sensor heads and power meter. Directional couplers are used to sample a portion of the reflected and transmitted power signals which is routed to crystal (diode) detectors.

While the cavity's transmitted power signal is amplified and conditioned in the PLL module, it is also routed to the PMM via a $10 \mathrm{~dB}$ coupler and likewise interfaced to a power meter sensor head and power meter.

\section{High Power Amplifier Switching Network}

The High Power Amplifier Switching Network provides the capability to switch the LLRF drive signal between a low power (LP) and high power (HP) amplifier. The integrated LP amplifier provides a more consistent and simple cable calibration capability. It also permits cable calibration to be performed without having the external Dewar shielding lid moved into place, restricting access to the Dewar top plate, as would be required if the interlocked HP amplifier was used.

This module contains two SPDT RF switches that route the RF signal to the selected amplifier input and from the selected amplifier output to the common RF cable to the Dewar. The high power request is activated by a switch that interfaces to a circuit which controls switch timing, preventing the high power switch from switching under full load. It ensures that the LLRF drive signal to the HP amplifier is switched off for two or more seconds prior to switching the amplifier network output from the HP to the LP amplifier. This allows the cavity field to decay and reflected power to be absorbed by the HP amplifier's internal protection system, before the output of the LP amplifier is connected to the cavity. The circuit that operates the switches requires the presence of an HP enable signal from the PSS interlock system. This signal is not present unless the Dewar shielding lid interlock is satisfied and no radiation is detected on the area monitors.

A high power circulator and load are connected to the output of the high power switch, serving as an isolator and providing additional protection for the amplifiers. It also ensures a better VSWR match for the forward and reflected power measurement circuits. Between this circulator and the cavity is a $20 \mathrm{~dB}$ dual-directional coupler that is used to couple samples of the incident and reflected power to the Power Measurement Module.

The HP amplifier provides a $500 \mathrm{~W}$ output over a frequency range of $1.27-1.31 \mathrm{GHz}$, with a minimum small signal gain of $58 \mathrm{~dB}$, and a maximum input and output VSWR of 2.0. The low power amplifier provides $30 \mathrm{~dB}$ gain and $28 \mathrm{dBm}$ output power over the frequency range $0.7-4.2 \mathrm{GHz}$. An input attenuator of $10 \mathrm{~dB}$ is required in order to match the input signal level required by the HP amplifier with that of the LP amplifier. 


\section{Signal Conditioning Module}

The signal from the diode detectors, which have a $23 \mathrm{dBm}$ maximum input level, are routed to the Signal Conditioning Module, which utilizes low-noise instrumentation amplifiers to electronically isolate and condition these signals, and provides the capability of applying up to $54 \mathrm{~dB}$ of gain to the output voltage, thereby scaling the maximum of the crystal detector square-law range $(-50$ to $-16 \mathrm{dBm})$ to the level of a few volts. The software warns the operator if this voltage is exceeded during a decay measurement.

\section{Control and Data Acquisition Platform}

The data acquisition system utilizes a National Instruments 8-slot PXI crate housing a Pentium-based $2 \mathrm{GHz}$ controller with Windows XP OS. Control and DAQ $\mathrm{SW}$ is written in the LabView programming language. Connectivity to the RF system is provided via USB, GPIB, serial, and PXI interfaces. A National Instruments PXI-6229 M-series multi-function DAQ module provides 32 16-bit analog input channels, 4 16-bit analog output channels, and up to 48bits of Digital I/O. These input/output channels provide control interfaces for the vector modulator, attenuator, and crystal detectors, and also accommodate analog input of various test environment parameters, such as Dewar pressure and radiation values. The GPIB interface provides control of power meters, RF source, and Dewar/cavity thermometry. The extensibility inherent in the PXI architecture easily accommodates future expansion or system modification options.

\section{DATA ACQUISITON \& CONTROL SOFTWARE}

The software for RF control and data acquisition was developed using the National Instruments LabView programming environment. The software, as originally developed by and used extensively at Jefferson Lab, provides automation for many of the tasks required to perform an RF test of superconducting cavities, and was used in several prototype and production testing efforts at Jefferson Lab. The original software was modified to operate with the specific instruments and electronics at Fermilab.

A finite state machine [4] is used as the central state machine. The following tasks are automated and can be performed by the operator at any time :

- instrument initialization

- cable calibration routine

- cavity field decay measurement

- CW measurement

- pulsed RF operation

- phase optimization

- $\quad$ auto-step and measure function.

The software provides complete control of RF amplitude and phase, measures and records field-emission radiation, Dewar pressure and bath temperature, provides real-time plotting of $\mathrm{Q}_{0}$ vs $\mathrm{E}$ and radiation, and calculates errors associated with all measured and calculated (derived) quantities.

All measured data is written to a tab delimited text file. A separate cable calibration log file is updated after each execution of the cable calibration routine, and allows historical calibration data to be examined in order to investigate system performance changes.

\section{INITIAL SYSTEM PERFORMANCE}

Individual system modules were tested independently once completed to ensure that signal levels throughout their circuits were appropriate (larger than expected signal attenuation indicating potential cable, connector, or component failure). Module functionality (switch, vector modulator, digital attenuator operation, etc.) was verified. The modules were then integrated and tested as a system, using a Cu cavity at $1.5 \mathrm{GHz}$ with a $\mathrm{Q}$ of $10^{4}$. The LLRF system locked onto the cavity and tracked cavity frequency well. The DAQ \& control software was tested and refined, and operation with the Cu cavity successfully demonstrated.

\section{SUMMARY}

The RF system design and fabricated for testing of ILC cavities at the Fermilab VTS is based upon a proven and easily-modified design and utilizes commercial components to ensure reliability, ease of manufacture and repair, and stable performance. Limited initial testing has shown that the system performs as expected, with minimal refinement expected before the system can be used for production scale testing.

\section{ACKNOWLEDGMENTS}

The authors are deeply indebted to the technical staff of the Fermilab Technical Division Test \& Instrumentation department for their invaluable assistance in the fabrication, assembly, and testing of the assorted RF modules and components.

\section{REFERENCES}

[1] J. P. Ozelis et al., "Design and Commissioning of Fermilab's Vertical Test Stand for ILC SRF Cavities", paper WEPMN106, this conference

[2] C. Reece, T. Powers, P. Kushnick, "An Automated RF Control and Data Acquisition System for Testing Superconducting RF Cavities", Proceedings of the 1991 Particle Accelerator Conference, v.3, pp15081510, San Francisco, CA, 1991

[3] T. J. Powers, "Theory and Practice of Cavity RF Test Systems”, Proceedings of the 12th International Workshop on RF Superconductivity”, Ithaca NY, July 2005.

[4] Black, P. E., ”Finite State Machine Definition”, NIST, http://www.nist.gov/dads/HTML/finiteStateMachine. html 